\title{
«Der Mensch ist keine Maschine»
}

\section{Daniel Lüthi}

Freier Journalist und Fotograf, Medientrainer, Bern

Welcher Gegensatz: Wir sitzen in einem gemütlichen Chalet über dem Tal und geniessen den Weitblick hinunter ins Unterwallis und hinauf Richtung Grimselund Furkapass. Gleichzeitig tauchen wir ein in die Enge von Institutionen, die einst "Anstalt»" hiessen. Wir sehen grüne Wiesen und Wälder, Berge und rustikale Hausdächer im Sonnenlicht. Und wir erinnern uns an die dunklen Zeiten der Psychiatrie in diesem Land.

\section{Hospitalismus und Befreiung}

«Menschen waren jahrzehntelang eingeschlossen in armseligen, erbärmlichen Zellen», erzählt Daniel Hell, «Vorurteile waren damals zugleich Urteile. Das kann man sich heute gar nicht mehr vorstellen.» Damals ist nicht lange her - das war etwa 1970, zum Beispiel in der Klinik Burghölzli in Zürich. «Das war die Zeit des Hospitalismus», erklärt Hell, damals junger (und staunender!) Assistenzarzt. «In der geschlossenen Abteilung, in der ich damals arbeitete, betrug die durchschnittliche Hospitalisationszeit 16 Jahre, es gab dort aber auch Menschen, die über 40 Jahre lang eingesperrt blieben.» Einmal im Jahr machte diese Abteilung einen Ausflug mit dem Car. Eine Gelegenheit, Patienten nicht als zu behandelnde Fälle zu sehen, sondern als persönliches Gegenüber zu erleben. Daniel Hell erkannte Chancen und entwickelte neue Ideen. Er führte ein erstes Ferienlager mit den chronisch Kranken dieser geschlossenen

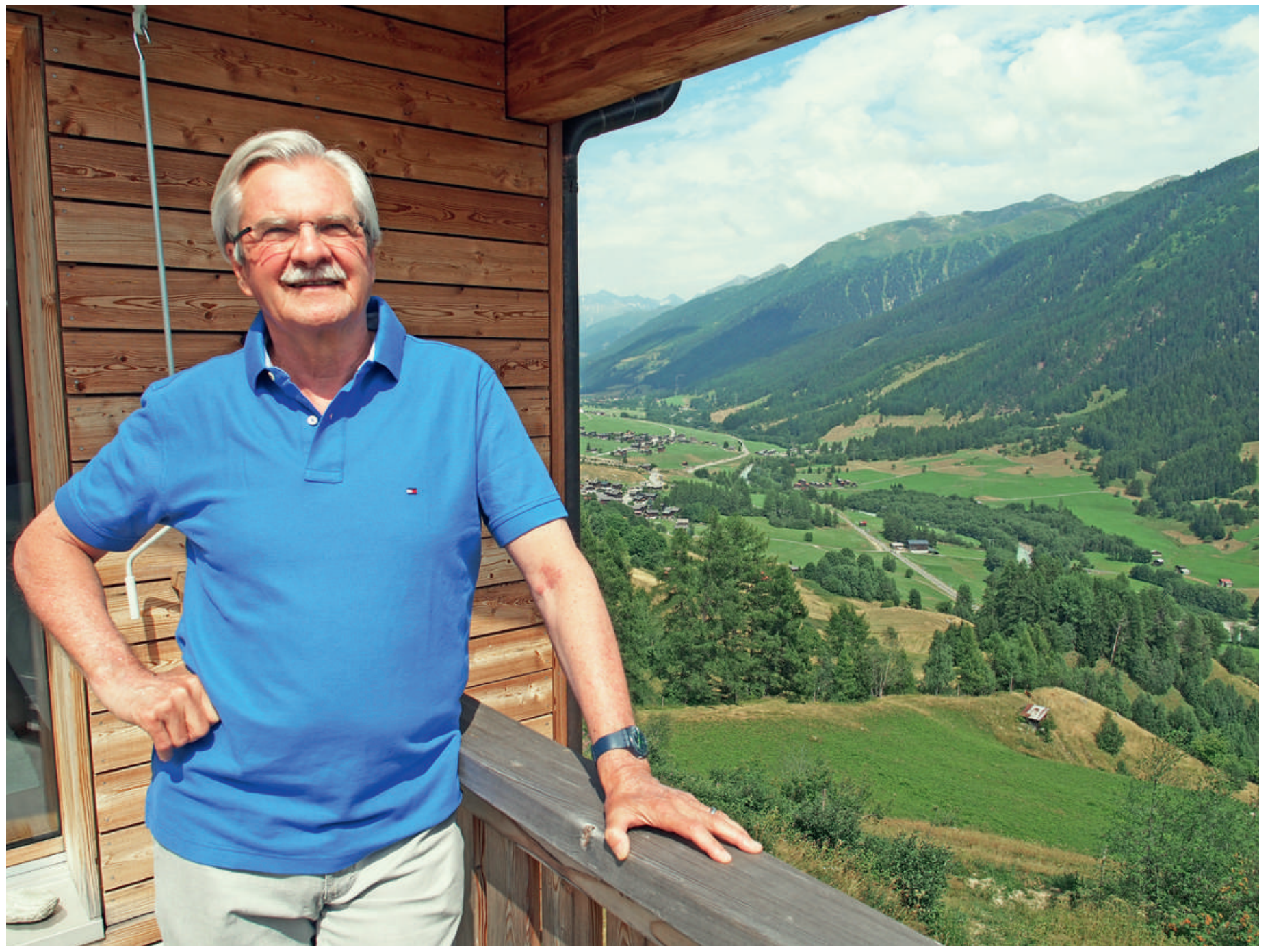


Abteilung durch, «was in der Folge zur Öffnung dieser Station führte. Ein betroffener Schizophreniekranker sagte mir damals, als er vom Plan der Öffnung hörte: 'Sie sind ja verrückt.'» Hell lacht, und plötzlich sind die Gegensätze wieder ganz nahe beieinander: hell und dunkel, Himmel und Hölle. Sein Nachname ist Programm. Daniel Hell weiss das.

Und auch diejenigen, die ihn damals kritisierten, wissen: Diese Öffnung war ein Befreiungsschlag - Teil einer Reformbewegung, die damals in der Psychiatrie um sich griff. «Es entwickelte sich eine neue Einstellung, eine offenere Haltung den Patientinnen und Patienten gegenüber.» Daniel Hell ist in der Schweizer Psychiatrie ein Pionier, eine prägende Figur seines Fachs. Die Beziehung zum Patienten wurde ins Zentrum gerückt, dies sei der Kern der neuen Bewegung gewesen, sagt er. «Wir wollten ambulante Behandlungen ausbauen und mit stationären besser vernetzen. Vor allem aber wollten wir das Verständnis für psychisch kranke Menschen fördern. Ganz nach dem Motto von Manfred Bleuler: 'Bleibt beim Kranken'. Auch ich bin überzeugt: Die Beziehungsgestaltung ist unser Hauptinstrument.»

Diese Sichtweise jedoch sei zunehmend verdrängt worden, bedauert Hell, «Die Psychiatrie wird immer mehr auf wirtschaftliche und neurologische Aspekte eingeengt, auf Geld und Gehirn - auf das, was von aussen als Objekt erfasst werden kann. Was heute zählt, ist das Fassbare, das Messbare, das Analysierbare. Das wird in der Ausbildung gefördert, und das macht $\mathrm{zu}$

\section{«Es bleibt immer etwas Unhintergehbares.»}

einem grossen Teil auch den technischen Fortschritt in der Medizin aus.» Gerade die Psychiatrie aber sei vielschichtiger, und das menschliche Leben sowieso, «fast nichts ist linear und es gibt viele Faktoren, die es zu berücksichtigen gilt, neben neurowissenschaftlichen insbesondere auch psychosoziale. Der Mensch ist keine Maschine.» Deshalb könne ihm die Medizin mit Maschinen und Medikamenten allein auch nicht gerecht werden: «Mit dem Apparatenblick können wir den Menschen nicht ganz erfassen.»

\section{Der Grenzgänger}

Die Grenzen zwischen Arzt und Philosoph sind durchlässig bei Daniel Hell. «Ich bin ein Grenzgänger», sagt er selber, «Das Geistes- und Kulturwissenschaftliche hat mich immer gleichermassen fasziniert wie die Naturwissenschaft.» Vieles in der Medizin sei nachweisbar, durch Studien belegt, ja, «aber es bleibt immer auch etwas Unhintergehbares». Ein spezielles

\section{Daniel Hell}

Prof. Dr. med. Daniel Hell wurde 1944 in Uzwil geboren. Die Schulen besuchte er in Zürich und Basel, dort absolvierte er auch sein Medizinstudium. Dieses schloss er $1971 \mathrm{mit}$ dem Staatsexamen ab. Nach Assistenzjahren in verschiedenen Institutionen war er 1977 bis 83 Oberarzt an

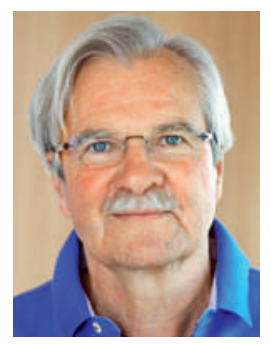
der psych. Universitätsklinik Zürich, bis 1991 dann Chefarzt der kantonalen psych. Klinik Breitenau in Schaffhausen. 1991 wurde er von der Uni Zürich zum Ordentlichen Professor für klinische Psychiatrie ernannt, bis 2009 war er ärztlicher bzw. klinischer Direktor der psychiatrischen Uniklinik Zürich, seit 2009 ist er emeritiert. Von da an bis 2014 war er Leiter des Kompetenzzentrums "Depression und Angst" an der Privatklinik Hohenegg in Meilen, seit diesem Jahr ist er dort im Stiftungsrat. Er engagiert sich ehrenamtlich in der Sozialpsychiatrie. Daniel Hell ist verheiratet, Vater und Grossvater. Er lebt mit seiner Frau in Erlenbach an der Sonnenküste des Zürichsees und immer öfter auch oberhalb von Blitzingen im Goms.

Wort. Hell meint das Unfassbare, das Geheimnisvolle, hinter das auch die Wissenschaft nicht kommt. Etwas zudem, das man nicht hintergehen kann im Sinne von täuschen oder betrügen. "Früher sagte man dem Seele», sagt Hell. Ihn auch als Seelsorger zu sehen, sei darum nicht falsch. Grenzen gebe es so oder so: «Ich kann und will das letzte Geheimnis nicht lüften.»

So spricht einer, der in seinem beruflichen Leben zu wesentlichen Themenfeldern - insbesondere Depression und Angst - Wesentliches beigetragen hat und dafür immer wieder geehrt wurde. So dieses Jahr mit dem Buch Das eigene Leben - jemand sein dürfen, statt etwas sein müssen*. Es nennt sich "Denkschrift für Daniel Hell», wurde als Folge eines Symposiums zu seinem 70. Geburtstag herausgegeben - und wirkt wie ein Nachruf. Fachleute aus der Medizin, aber auch der Schriftsteller Adolf Muschg oder Altbundesrat Moritz Leuenberger haben Texte beigesteuert, durch die sie mit Daniel Hell in einen Dialog treten wollen. Der Begriff «Nachruf» amüsiert Hell. «Meine institutionelle Tätigkeit und meine akademische Lehrtätigkeit sind beendet», sagt er, «das schon. Aber ich spüre sehr viel Bewegtheit. Auch dieses Buch stimuliert mich mehr, als dass es mich bremsen würde. Es ist ein Geschenk, das ich nicht erwartet habe.» - «Macht es Sie stolz?» - «Stolz ist mir zu nahe am Hochmut - es macht mir grosse Freude.»

Gleich wie die Vorstellung, jetzt vermehrt ehrenamtliche Arbeit leisten zu können: sich für sogenannte Randständige einzusetzen, mit ihnen in sozialen Institutionen Kaffee zu trinken beispielsweise, und ihnen 
bei dieser Gelegenheit vielleicht auch als Psychiater ein Partner zu sein.

\section{Depression im Wandel}

Einer der Schwerpunkte im erwähnten Buch ist ein zentrales Thema in Daniel Hells beruflicher Karriere: Depression. «Ihre Kriterien sind in den letzten Jahrzehnten stark ausgeweitet worden», erklärt er. «Vor 100 Jahren war mit Depression vor allem die manischdepressive Erkrankung gemeint, davon war nur rund ein Prozent der Bevölkerung betroffen.» In der Mitte des 20. Jahrhunderts seien vermehrt depressive Neurosen diagnostiziert worden, die Rate der Betroffenen stieg auf fünf Prozent. «Später kamen rein depressive Episoden hinzu und die Depressionskriterien wurden auf das Vorliegen einiger Symptome reduziert. Heute gehen wir davon aus, dass bis ein Viertel der Bevölkerung an einer Depression leidet oder litt.»

\section{«Heute fehlt es an Zeit für die Patienten, und an Nachwuchs.»}

Problematisch an dieser Entwicklung sei der Kurzschluss von bestimmten Leidensformen auf das Vorliegen einer Krankheit, sagt Hell. "Man kann auch an Demütigungen und Konflikten leiden. Ab den 80er Jahren wurden die Diagnosen aber weitgehend unabhängig vom Kontext. Die Symptome selber wurden pathologisiert.» Parallel dazu seien Zeit- und Effizienzdruck grösser geworden - in der ganzen Gesellschaft, also auch in der Psychiatrie. Dies habe zu mehr Behandlungen geführt, gleichzeitig aber auch zu einem Mangel bei den Fachleuten: «Heute fehlt es an Zeit für die Patienten, und an Nachwuchs. Synergien von Ökonomie, digitalisierter Technik und naturwissenschaftlicher Empirie prägen die Psychiatrie.»

Früher haben laut Hell vor allem Schuldgefühle depressiven Menschen zu schaffen gemacht. «In hierarchischen, patriarchalischen Strukturen fühlten sich viele von Geboten oder von ihrem Über-Ich dominiert. Sie fühlten sich oft bereits wegen Kleinigkeiten schuldig und erlebten die Depression als Strafe. Heute, im forcierten Individualismus, herrscht das Ich-Ideal. Wer daran scheitert, schämt sich. Die Ansprüche - auch an uns selber - sind gewachsen, wir erwarten oft mehr, als wir tun können.» Eine Folge davon seien vermehrte Selbstwertprobleme, gerade bei depressiven Menschen. Im erwähnten Buch schreibt er dazu: «Der Umgang mit Scham ist für mich zur Nagelprobe in der therapeutischen Arbeit geworden. Es ist ein Selbst- gefühl, das die eigenen Grenzen - zwischen innen und aussen, zwischen Wunsch und Realität - schmerzhaft spüren lässt.»

\section{Ängste - auch eigene}

Ein zweites Spezialgebiet von Daniel Hell ist die Angst in ihren verschiedenen Ausprägungen. «Zuerst einmal: Angst ist ein wesentliches Gefühl. Wer sie spürt, spürt sich selber», sagt er dazu. «Es ist aber auch ein Erlebnis, das einengt. Und dazu ein Sensor, der vor Gefahren warnt, also auch eine schützende Funktion hat.» Ängste hätten den gegenteiligen Effekt einer Depression. «Eine Depression lähmt, führt zum Stillstand. Ängste aktivieren - Herz und Lunge beispielsweise arbeiten mehr. Wenn sich Ängste in der Vorstellung aber hochschaukeln, können sie übermächtig und behindernd werden - und in die Krankheit führen.» Kürzlich sei er beim Wandern in ein Gewitter geraten, erzählt er, und ja, «ich habe schon etwas wie Angst gespürt.» Keine Rede also davon, dass er selber, der so viele therapiert hat, gegen Ängste immun ist. «Ich kenne selber verschiedene Angstformen, darunter die Scham-Angst. Auch ich will mich nicht blossstellen, auch ich brauche Zuspruch, Anerkennung und Vertrauen.» Und die grosse, die letzte Angst, diejenige vor dem Tod - kennt er sie? Die Situation, als professioneller Fragender wieder einmal auf der anderen Seite zu sitzen und mit persönlichen Fragen konfrontiert zu sein, macht Hell keine Mühe, eher Spass. «Früher dachte ich fast ausschliesslich ans Leben", antwortet er nach einem langen Blick talabwärts. «Heute auch ab und zu ans Sterben. Und ja: Das macht mir schon Sorgen.» Als Arzt wisse er sehr wohl, dass in seinem Hirn jederzeit eine Ader platzen könnte, «dann wäre ich von einer Sekunde zur nächsten ein ganz anderer Mensch oder einfach weg."

Er verschliesse sich auch persönlichen Fragen von $\mathrm{Pa}$ tienten nicht, sagt Hell. «Ein Therapeut muss sich verstehend, einfühlend einbringen und dem Patienten als Person begegnen. Selber muss er kongruent sein.» Will heissen: ein Mensch, keine Maschine.

\section{Die nächste Begegnung mit...}

Am Ende jeden Monats stellt die Schweizerische Ärztezeitung eine Persönlichkeit vor, die sich im Gesundheitswesen engagiert. Im Oktober schildert Daniel Lüthi seine Begegnung mit Zaheer Ahmed, Mitarbeiter des Patientenbegleitdienstes am UniversitätsSpital Zürich. 